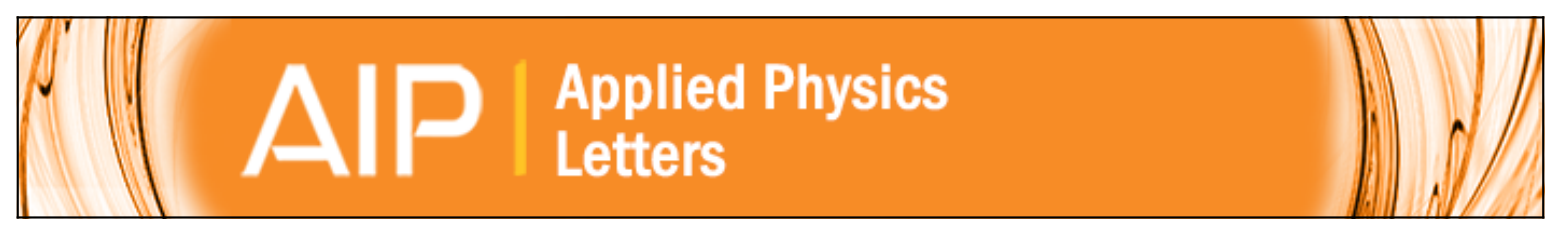

\title{
Direct and converse effect in magnetoelectric laminate composites
}

Kyung-Hoon Cho and Shashank Priya

Citation: Applied Physics Letters 98, 232904 (2011); doi: 10.1063/1.3584863

View online: http://dx.doi.org/10.1063/1.3584863

View Table of Contents: http://scitation.aip.org/content/aip/journal/apl/98/23?ver=pdfcov

Published by the AIP Publishing

AlP Re-register for Table of Content Alerts 


\title{
Direct and converse effect in magnetoelectric laminate composites
}

\author{
Kyung-Hoon $\mathrm{Cho}^{\mathrm{a})}$ and Shashank Priya ${ }^{\mathrm{b})}$ \\ Center for Energy Harvesting Materials and Systems (CEHMS), Center for Intelligent Material Systems \\ and Structures (CIMSS), Virginia Tech, Blacksburg, Virginia 24061, USA
}

(Received 24 February 2011; accepted 11 April 2011; published online 10 June 2011)

\begin{abstract}
In this letter, we analyze the direct and converse effect in laminate composites of magnetostrictive and piezoelectric materials. Our results deterministically show that direct magnetoelectric (ME) effect is maximized at antiresonance frequency while the converse ME effect is maximized at resonance frequency of the laminate composite. We explain this phenomenon by using piezoelectric constitutive equations and combining it with resonance boundary conditions. The dominant factor controlling the position of peak ME coefficient was found to be frequency dependent capacitance of piezoelectric layer. This study will provide guidance toward the development of magnetic field sensors based on direct effect and communication components based on converse effect. (C) 2011 American Institute of Physics. [doi:10.1063/1.3584863]
\end{abstract}

Conversion of magnetic field to electric polarization [direct magnetoelectric (ME) effect or DME effect] or electric field to magnetization (converse ME effect or CME effect) is attractive for various applications such as magnetic field sensors, tunable phase shifters, filters, and optical components. ${ }^{1-4}$ It has been shown both theoretically and experimentally that ME effect could be several orders of magnitude larger in piezoelectric-magnetostrictive laminate composites (2-2 connectivity) that exploit product property as compared to single phase materials. ${ }^{5,6}$ Within the composite family, 2-2 connectivity has been shown to be superior to that of 3-0 and 3-1 connectivities. ${ }^{7-9}$ In addition to design of 2-2 laminate composites, several researchers have reported giant enhancement in the magnitude of ME coefficient at the electromechanical resonance. Thus, a combination of 2-2 laminate structure operating at resonance presents a tremendous opportunity for configuring the magnetoelectric devices. However, we noticed a serious discrepancy about the reported peak position of DME and CME. The results reported in literature show that both DME (Ref. 2) and CME (Refs. 10-13) effect of laminate ME composites can be maximized at electromechanical resonance (EMR) frequency of P-layer. We deterministically prove that this is not the case. We report that DME effect in laminate ME composites is maximized at antiresonance frequency $\left(f_{\mathrm{a}}\right)$ while the CME effect is maximized at EMR frequency $\left(f_{\mathrm{r}}\right)$ of piezoelectric layer.

DME and CME effects in the laminate composites consisting of piezoelectric layer (P-layer) and magnetostrictive layer (M-layer) can be represented by following schematics:

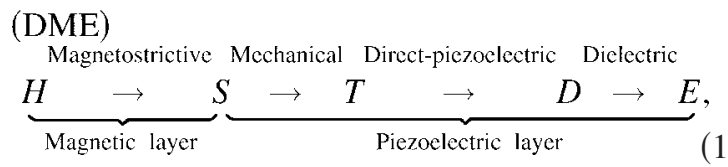

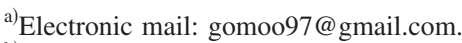

${ }^{b)}$ Electronic mail: spriya@vt.edu.
}

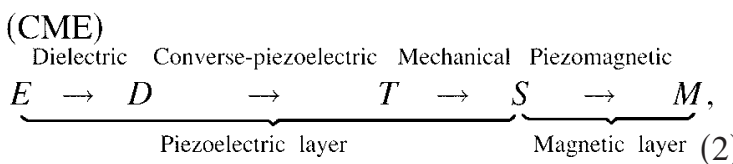

where $H$ is magnetic field, $S$ is mechanical strain, $T$ is mechanical stress, $D$ is electric displacement, $E$ is electric field, and $M$ is magnetization. For DME effect, input magnetic field $H$ generates a strain $S$ in M-layer by magnetostriction and this strain is transferred to P-layer through elastic coupling. Next, a mechanical stress $T$ is generated in P-layer resulting in surface charge density $(D)$ or polarization by direct piezoelectric effect. Due to this charge separation, output voltage or electric field is induced in P-layer. In the case of CME effect, input electric field $E$ induces polarization in P-layer resulting in stress and strain by converse piezoelectric effect. This strain is transferred to M-layer by elastic coupling inducing magnetization $M$ through piezomagnetism. Due to the fact that DME or CME in laminate ME composites is a product property of individual P- and M-layer, ${ }^{1}$ the ME response of composites is strongly dependent on the physical properties of P-layer. DME voltage coefficient of longitudinally magnetized and transversely poled system (L-T mode) is given as ${ }^{1,14}$

$$
\begin{aligned}
\alpha_{\mathrm{E}, 31}= & \frac{d E_{3, \mathrm{ac}}}{d H_{1, \mathrm{ac}}}=\frac{n q_{11} g_{31}}{n S_{11}^{\mathrm{E}}\left(1-k_{31}^{2}\right)+(1-n) S_{11}^{\mathrm{H}}} \\
& \times \frac{1}{\exp \left(\tan \delta^{\prime}+\tan \theta^{\prime}+\frac{C-C_{\mathrm{f}}}{C_{\mathrm{f}}}\right)},
\end{aligned}
$$

where $H_{1}$ is longitudinally applied magnetic field along 1 axis, $E_{3}$ is electric field generated from the P-layer perpendicular to $H_{1}$ direction ( 3 axis), $n$ is volume fraction of M-layer, $q_{11}$ is piezomagnetic coefficient of M-layer, $g_{31}$ is piezoelectric voltage coefficient of P-layer, $k_{31}$ is electromechanical coupling coefficient of P-layer, $S_{11}^{\mathrm{E}}$ and $S_{11}^{\mathrm{H}}$ are elastic compliance of $\mathrm{P}$ - and M-layers, respectively, and $\tan \delta^{\prime}$, $\tan \theta^{\prime}$, and $\left(C-C_{\mathrm{f}}\right) / C_{\mathrm{f}}$ are dielectric loss, piezoelectric loss, and relative capacitance change in P-layer, respectively. The exponential term in Eq. (3) represents a frequency dependent loss factor of dynamic DME response. Equation (3) predicts that high $\alpha_{\mathrm{E}, 31}$ value can be obtained when the P-layer has 


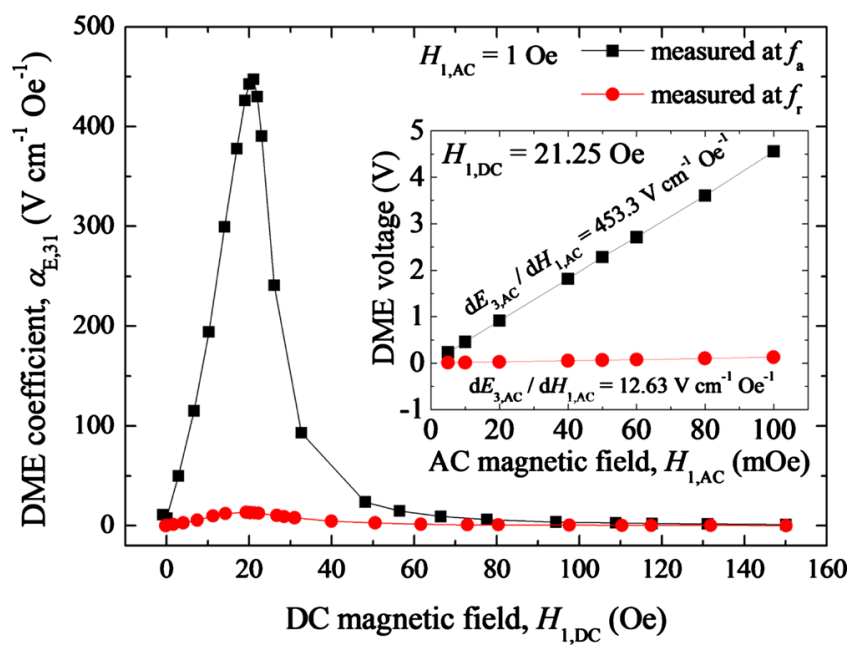

FIG. 1. (Color online) DME coefficient $\left(\alpha_{\mathrm{E}, 31}\right)$ of the MP laminate measured at resonance frequency $\left(f_{\mathrm{r}}\right)$ and antiresonance frequency $\left(f_{\mathrm{a}}\right)$ under varying dc magnetic field $\left(H_{1, \mathrm{dc}}\right)$. Inset shows DME voltage output of the MP laminate measured at $f_{\mathrm{r}}$ and $f_{\mathrm{a}}$ under various ac magnetic field $\left(H_{1, \text { ac }}\right)$ and constant $H_{1, \mathrm{dc}}$ of 21.25 Oe.

low $S_{11}^{\mathrm{E}}$, high $k_{31}$, high $g_{31}$ and low dielectric and piezoelectric losses at a given frequency.

Using these considerations, we fabricated a 2-2 laminate ME composite consisting of M- and P-layers (MP laminate). Piezoelectric composition $0.05 \mathrm{~Pb}\left(\mathrm{Mn}_{1 / 3} \mathrm{Sb}_{2 / 3}\right) \mathrm{O}_{3}-0.95 \mathrm{~Pb}$ $\times\left(\mathrm{Zr}_{0.52} \mathrm{Ti}_{0.48}\right) \mathrm{O}_{3}$ was synthesized as the P-layer by solid state reaction method and shaped in to a 31-mode plate (27 $\times 5 \times 1 \mathrm{~mm}^{3}$ ). After poling this P-layer, $46-\mu \mathrm{m}$-thick Metglas (2605SA1, Metglas Inc., USA) sheet (M-layer) was attached by using epoxy (West System, USA) on top and bottom of the P-layer. The DME effect was measured in L-T mode configuration. The MP laminate was placed in the center of the Helmholtz coil $\left(H_{1, \text { ac }}\right)$ which itself was located at the center of electromagnet $\left(H_{1, \mathrm{dc}}\right)$. The voltage induced on the laminate was monitored using lock-in amplifier. To observe the CME effect, electric field $\left(E_{3, \text { ac }}\right)$ was applied to the P-layer and the voltage induced on Helmholtz coil was moni-

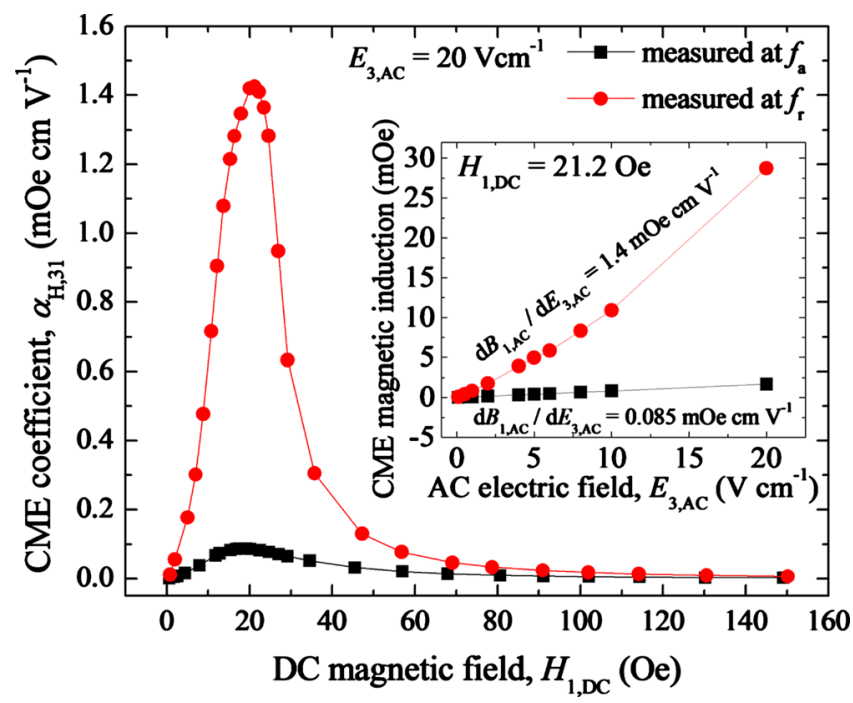

FIG. 2. (Color online) CME coefficient $\left(\alpha_{\mathrm{H}, 31}\right)$ of the MP laminate measured at resonance frequency $\left(f_{\mathrm{r}}\right)$ and antiresonance frequency $\left(f_{\mathrm{a}}\right)$ under varying dc magnetic field $\left(H_{1, \mathrm{dc}}\right)$. Inset shows CME magnetic induction $\left(B_{1, \text { ac }}\right)$ of the MP laminate measured at $f_{\mathrm{r}}$ and $f_{\mathrm{a}}$ under various ac electric field $\left(E_{3, a c}\right)$ and censtant $H_{1, \mathrm{ce}}$ of $21,2, \mathrm{Qe}$

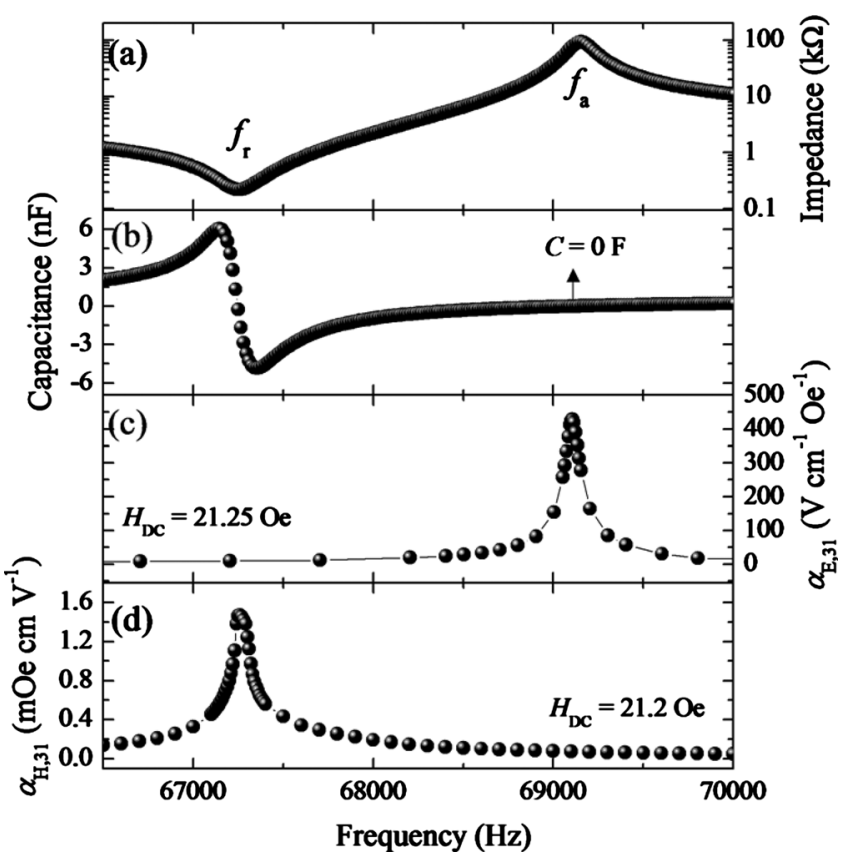

FIG. 3. (a) Impedance versus frequency of the P-layer, (b) capacitance versus frequency of the P-layer, (c) $\alpha_{\mathrm{E}, 31}$ versus $H_{1, \text { ac }}$ frequency, and (d) $\alpha_{\mathrm{H}, 31}$ versus $E_{3, \text { ac }}$ frequency characteristic of the MP laminate. The result clearly shows that $\alpha_{\mathrm{E}, 31}$ is maximized near $f_{\mathrm{a}}$ while $\alpha_{\mathrm{H}, 31}$ has the maximum value near $f_{\mathrm{r}}$ of the P-layer.

tored. This induced voltage was converted to magnetic induction $\left(B_{1, \text { ac }}\right)$.

Figure 1 shows the DME coefficient $\left(\alpha_{\mathrm{E}, 31}\right)$ of the MP laminate measured at $f_{\mathrm{r}}$ and $f_{\mathrm{a}}$ under varying dc magnetic field $\left(H_{1, \mathrm{dc}}\right)$. When the laminate was operated at $f_{\mathrm{a}}$, the $\alpha_{\mathrm{E}, 31}$ value was found to increase with increasing $H_{1, \mathrm{dc}}$ showing the maximum magnitude of $448 \mathrm{~V} \mathrm{~cm}^{-1} \mathrm{Oe}^{-1}$ at $21.25 \mathrm{Oe}$ $H_{1, \mathrm{dc}}$, and then decreasing with increasing $H_{1, \mathrm{dc}}$. The tendency of $\alpha_{\mathrm{E}, 31}$ with variation in $H_{1, \mathrm{dc}}$ is related to the piezomagnetic coefficient $\left(q_{11}=\partial \lambda_{1} / \partial H_{1}, \lambda_{1}\right.$ : magnetostriction) of Metglas M-layer in Eq. (3) and the highest $q_{11}$ value is expected to occur at $H_{1, \mathrm{dc}}=21.25 \mathrm{Oe}$. However, at $f_{\mathrm{r}}$, the maximum $\alpha_{\mathrm{E}, 31}$ value $\left(13 \mathrm{~V} \mathrm{~cm}^{-1} \mathrm{Oe}^{-1}\right)$ was much smaller than that of $f_{\mathrm{a}}$ case. Inset of Fig. 1 shows DME output voltage measured at $f_{\mathrm{r}}$ and $f_{\mathrm{a}}$ with varying ac magnetic field $\left(H_{1, \text { ac }}\right)$ at constant $H_{1, \text { dc }}$ of 21.25 Oe. By definition of DME coefficient in Eq. (3), we obtained $\alpha_{\mathrm{E}, 31}$ values $\left(453.3 \mathrm{~V} \mathrm{~cm}^{-1} \mathrm{Oe}^{-1}\right.$ in $f_{\mathrm{a}}$ case and $12.63 \mathrm{~V} \mathrm{~cm}^{-1} \mathrm{Oe}^{-1}$ in $f_{\mathrm{r}}$ case) given by the slope as shown in the inset of Fig. 1. The calculated values were found to match well with the measured values in Fig. 1.

Next, we measured the CME coefficient $\left(\alpha_{\mathrm{H}, 31}\right.$ $\left.=d B_{1, \mathrm{ac}} / d E_{3, \mathrm{ac}}\right)$ of laminate both at $f_{\mathrm{r}}$ and $f_{\mathrm{a}}$ with varying $H_{1, \mathrm{dc}}$ as shown in Fig. 2. The trend of $\alpha_{\mathrm{H}, 31}$ as a function of $H_{1, \mathrm{dc}}$ was found to be similar to that of $\alpha_{\mathrm{E}, 31}$ in Fig. 1, indicating that CME effect is also dependent on the $q_{11}$ of M-layer. However, unlike DME effect, the maximum $\alpha_{\mathrm{H}, 31}$ value (1.42 mOe cm V$~^{-1}$ ) occurring at $H_{1, \mathrm{dc}}$ of $21.2 \mathrm{Oe}$ was much higher at $f_{\mathrm{r}}$ than that at $f_{\mathrm{a}}\left(0.086 \mathrm{mOe} \mathrm{cm} \mathrm{V} \mathrm{V}^{-1}\right)$. Inset of Fig. 2 shows CME magnetic induction measured under varying ac electric field $\left(E_{3, \text { ac }}\right)$ at constant $H_{1, \mathrm{dc}}$ of 21.2 Oe. Calculated $\alpha_{\mathrm{H}, 31}$ values from the slope were in excellent agreement with the measured values in Fig. 2. In both DME and CME cases, a huge difference in ME coefficient value was observed between $f_{\mathrm{r}}$ and $f_{\mathrm{a}}$ operations. 
TABLE I. Measured or derived material parameters of $0.05 \mathrm{~Pb}\left(\mathrm{Mn}_{1 / 3} \mathrm{Sb}_{2 / 3}\right) \mathrm{O}_{3}-0.95 \mathrm{~Pb}\left(\mathrm{Zr}_{0.52} \mathrm{Ti}_{0.48}\right) \mathrm{O}_{3}$ piezoelectric composition.

\begin{tabular}{ccccc}
\hline \hline $\begin{array}{c}d_{31} \\
\left(10^{-12} \mathrm{C} \mathrm{N}^{-1}\right)\end{array}$ & $\begin{array}{c}h_{31} \\
\left(10^{8} \mathrm{~V} \mathrm{~m}^{-1}\right)\end{array}$ & $\begin{array}{c}\varepsilon_{33}^{\mathrm{T}} \\
\left(10^{-9} \mathrm{~F} \mathrm{~m}^{-1}\right)\end{array}$ & $\begin{array}{c}\varepsilon_{33}^{\mathrm{S}} \\
\left(10^{-9} \mathrm{~F} \mathrm{~m}^{-1}\right)\end{array}$ & $\begin{array}{c}s_{11}^{\mathrm{E}} \\
\left(10^{-12} \mathrm{~m}^{2} \mathrm{~N}^{-1}\right)\end{array}$ \\
\hline-63.85 & -14.45 & 5.12 & 2.64 & 9.95 \\
\hline
\end{tabular}

In order to confirm the accurate frequency where DME and CME effects exhibit peak, we measured $\alpha_{\mathrm{E}, 31}$ and $\alpha_{\mathrm{H}, 31}$ of the laminate as a function of applied frequency. It can be clearly seen from Fig. 3 that $\alpha_{\mathrm{E}, 31}$ value is maximized at $f_{\mathrm{a}}$ while $\alpha_{\mathrm{H}, 31}$ value is maximized at $f_{\mathrm{r}}$ of P-layer. There was no trace of enhancement of $\alpha_{\mathrm{E}, 31}$ value near $f_{\mathrm{r}}$ or enhancement of $\alpha_{\mathrm{H}, 31}$ value near $f_{\mathrm{a}}$. We attempt to explain the reason for this observation by invoking the piezoelectric effect in P-layer. By considering direct piezoelectric (DP) and converse piezoelectric (CP) energy conversion of P-layer in schematics (1) and (2) (Piezoelectric layer parts), following equations can be obtained:

$$
\begin{aligned}
& \mathrm{DP}:\left|\left(\frac{\partial T_{1}}{\partial S_{1}}\right)_{\mathrm{D}} \times\left(\frac{\partial D_{3}}{\partial T_{1}}\right)_{\mathrm{E}} \times\left(\frac{\partial E_{3}}{\partial D_{3}}\right)_{\mathrm{S}}\right|=\left|\frac{d_{31}}{S_{11}^{\mathrm{D}} \times \varepsilon_{33}^{\mathrm{S}}}\right|, \\
& \mathrm{CP}:\left|\left(\frac{\partial D_{3}}{\partial E_{3}}\right)_{\mathrm{T}} \times\left(\frac{\partial T_{1}}{\partial D_{3}}\right)_{\mathrm{S}} \times\left(\frac{\partial S_{1}}{\partial T_{1}}\right)_{\mathrm{E}}\right|=\mid \varepsilon_{33}^{\mathrm{T}} \times h_{31} \\
& \quad \times S_{11}^{\mathrm{E}} \mid,
\end{aligned}
$$

where $S_{11}^{\mathrm{D}}$ is elastic compliance of P-layer under constant electric displacement, $\varepsilon_{33}^{\mathrm{S}}$ is dielectric permittivity of P-layer under constant strain, and $h_{31}$ is piezoelectric stiffness coefficient of P-layer. Equations (4) and (5) represent the figureof-merit for piezoelectric conversion efficiency of materials demonstrating that high piezoelectric strain coefficient, low compliance and low dielectric permittivity are appropriate for the efficient DP conversion while high dielectric permittivity, high piezoelectric stiffness coefficient and high compliance are effective for the $\mathrm{CP}$ conversion. Note that dielectric permittivity is the frequency dependent term in Eqs. (4) and (5) and this term occurs in denominator for the DP conversion and in numerator for the CP conversion. The impedance $(Z)$ of P-layer in Fig. 3(a) is given by following equation:

$$
Z=\left\{i \omega C\left[k_{31}^{2} \times \frac{\tan \Gamma}{\Gamma}+1-k_{31}^{2}\right]\right\}^{-1},
$$

where $\omega=2 \pi f, C$ is capacitance, $\Gamma=(\omega l / 2) \sqrt{\rho S_{11}^{\mathrm{E}}}, l$ is length of P-layer, and $\rho$ is density of P-layer. The $f_{\mathrm{r}}$ is obtained by substituting $\cos \Gamma=0$ and this solution reveals a minimized $Z$ value in Eq. (6). Due to the minima in $Z$ value, ac can easily flow through the P-layer and $C$ value is maximized at $f_{\mathrm{r}}$ as seen in Fig. 3(b). On the other hand, $f_{\mathrm{a}}$ is obtained by substituting $\tan \Gamma=-\left[\left(1-k_{31}^{2}\right) / k_{31}^{2}\right] \Gamma$, and this solution indicates a maximized $Z$ value in Eq. (6) representing a minimized $C$ value $(\sim 0 \mathrm{~F})$ in Fig. 3(b). Since $C$ is directly proportional to dielectric permittivity, the minimized $C$ value at $f_{\mathrm{a}}$ enhances the DP conversion in Eq. (4), thereby, maximizing DME effect in schematic (1). The maximized $C$ value at $f_{\mathrm{r}}$ enhances the $\mathrm{CP}$ conversion in Eq. (5) and CME effect in schematic
(2). Moreover, the right-hand part of Eq. (4) can be rewritten as

$$
\left|d_{31} / S_{11}^{\mathrm{E}}\left(1-k_{31}^{2}\right) \times \varepsilon_{33}^{\mathrm{S}}\right|
$$

and this equation is closely related to the

$$
g_{31} / S_{11}^{\mathrm{E}}\left(1-k_{31}^{2}\right)
$$

term in Eq. (3).

The figure-of-merits for the conversion efficiency in Eqs. (4) and (5), measured and derived from the material parameters in Table I were found to be $2.63 \times 10^{9} \mathrm{~V} \mathrm{~m}^{-1}$ and $7.36 \times 10^{-11} \mathrm{~m} \mathrm{~V}^{-1}$, respectively. These values indicate that $0.038 \mathrm{ppm}$ strain is required to generate $1.0 \mathrm{~V} \mathrm{~cm}^{-1}$ electric field through the DP conversion while only $0.00736 \mathrm{ppm}$ strain can be achieved by CP conversion when the same $1.0 \mathrm{~V} \mathrm{~cm}^{-1}$ electric field is applied to P-layer. Using the $\lambda$ magnitude of Metglas M-layer (27 ppm) and assuming the same elastic coupling factor for both DME and CME cases, we can expect that DME effect should be much stronger than the CME effect in our MP laminate, corresponding well with the large difference seen between $\alpha_{\mathrm{E}, 31}$ and $\alpha_{\mathrm{H}, 31}$ values observed in Figs. 1 and 2.

In summary, this study shows that the DME effect in laminate ME composite is maximized at $f_{\mathrm{a}}$ while the CME effect is maximized at $f_{\mathrm{r}}$. To date, prior research on resonant characteristics of piezoelectric or ME devices have focused on $f_{\mathrm{r}}$ of piezoelectric structure rather than $f_{\mathrm{a}}$. However, the results of this study provide guidance toward achieving better performance by taking $f_{\mathrm{a}}$ into account.

The authors gratefully acknowledge the financial support from DARPA Heterostructural Uncooled Magnetic Sensors (HUMS) program (Grant No. FA8650-09-1-7945).

${ }^{1}$ C.-W. Nan, M. I. Bichurin, S. Dong, D. Viehland, and G. Srinivasan, J. Appl. Phys. 103, 031101 (2008).

${ }^{2}$ G. Srinivasan, Annu. Rev. Mater. Res. 40, 153 (2010).

${ }^{3}$ M. Fiebig, J. Phys. D: Appl. Phys. 38, R123 (2005).

${ }^{4}$ C.-W. Nan, G. Liu, Y. Lin, and H. Chen, Phys. Rev. Lett. 94, 197203 (2005)

${ }^{5}$ S. Priya, R. Islam, S. Dong, and D. Viehland, J. Electroceram. 19, 149 (2007).

${ }^{6}$ R. Islam, Y. Ni, A. Khachaturyan, and S. Priya, J. Appl. Phys. 104, 044103 (2008).

${ }^{7}$ Y. Ni, S. Priya, and A. Khachaturyan, J. Appl. Phys. 105, 083914 (2009).

${ }^{8}$ C.-S. Park, C. Ahn, and S. Priya, Philos. Mag. 90, 4443 (2010).

${ }^{9}$ S.-C. Yang, C.-S. Park, K.-H. Cho, and S. Priya, J. Appl. Phys. 108, 093706 (2010).

${ }^{10}$ J. G. Wan, J.-M. Liu, G. H. Wang, and C.-W. Nan, Appl. Phys. Lett. 88, 182502 (2006).

${ }^{11}$ Y. Jia, S. W. Or, H. L. W. Chan, X. Zhao, and H. Luo, Appl. Phys. Lett. 88, 242902 (2006).

${ }^{12}$ Y. Wang, F. Wang, S. W. Or, H. L. W. Chan, X. Zhao, and H. Luo, Appl. Phys. Lett. 93, 113503 (2008).

${ }^{13}$ J.-P. Zhou, L. Meng, Z.-H. Xia, P. Liu, and G. Liu, Appl. Phys. Lett. 92, 062903 (2008).

${ }^{14}$ K.-H. Cho, C.-S. Park, and S. Priya, Appl. Phys. Lett. 97, 182902 (2010). 